The mean duration of symptoms was 62.4 months (minimum 1 month; maximum 40 years). From the evacuation request until effective journey patients waited a mean of 13.9 months (missing data in 37 patients). At arrival, only $25.9 \%$ of patients were or had been on corticosteroids and only $15.5 \%$ on conventional disease modifying antirheumatic drug (DMARD) treatment, versus $62 \%$ and $67.2 \%$, respectively, after assessment in Portugal (graphic). Even common and cheap drugs, like prednisolone and hydroxychloroquine were underprescribed before our assessment. Twelve percent of patients required biologics. Hospitalizations related to disease activity or complications were required in $43.1 \%$. Severe damage, measured by indication for orthopaedic, cardiothoracic or vascular surgery, need of chronic dialysis or long-term oxygen therapy and permanent neurologic deficits was present in $34.5 \%$ of the patients. Three patients died. Regarding infectious comorbidities, $12.1 \%$ of patients were diagnosed with tuberculosis, $8.6 \%$ had chronic hepatitis B infection and $12.1 \%$ had evidence of previous contact with hepatitis $B$ virus.

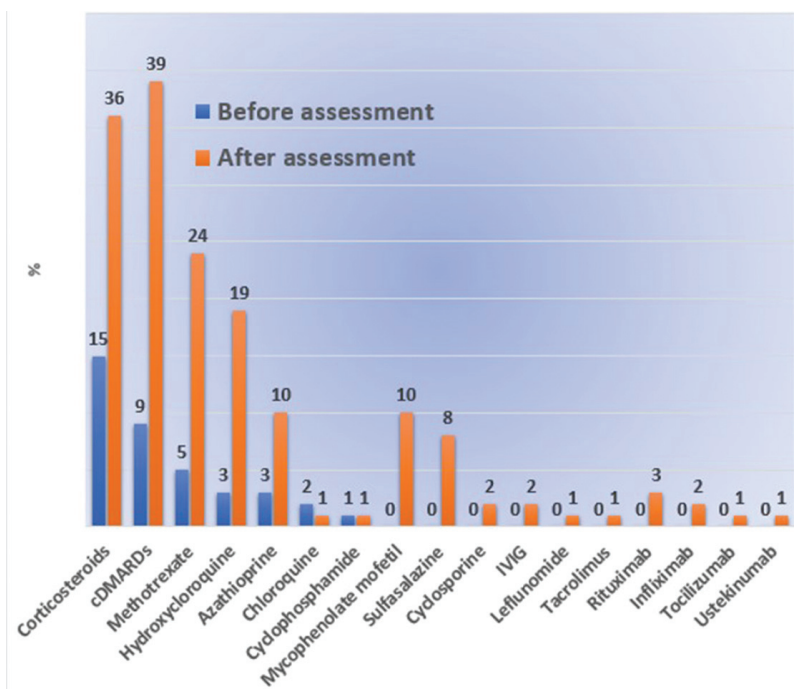

Abstract AB1271 - Figure 1

Conclusions: PALOP patients present with long-lasting and severe rheumatic diseases with chronic damage, due to lack of precise diagnosis, ineffective referrals and lack of appropriate treatment. They also frequently present with important infectious comorbidities and social needs that may delay treatment. Despite the obvious advantages of the evacuation of patients to a more resourceful country, we believe there is a need for identification of onsite barriers and improvement of local awareness on Rheumatic diseases and Rheumatology speciality.

Disclosure of Interest: None declared

DOI: 10.1136/annrheumdis-2018-eular.4955

\section{AB1272 INCREASING STRENGTHS OF EVIDENCE FOR ROLE OF NURSES IN THE MANAGEMENT OF CHRONIC INFLAMMATORY ARTHRITIS: RESULTS OF A SYSTEMATIC LITERATURE REVIEW}

V. Lion, M. Schirmer. Internal Medicine, Clinic II, MEDICAL UNIVERSITY OF INNSBRUCK, AUSTRIA, Innsbruck, Austria

Background: In 2011 EULAR first published European recommendations for the potential role of nurses in the management of patients with rheumatic diseases. ${ }^{1}$ Since then, the EULAR recommendations were well disseminated and positively evaluated both across Europe and the United States (US). ${ }^{2}$

Objectives: To assess new evidence for the role of nurses in the management of chronic inflammatory arthritis (CIA) obtained since the 2011 EULAR recommendations.

Methods: A systematic literature search was performed for the time between 1/ 2010 and 9/2016 based on the PRISMA guidelines, using the search strategies and eligibility criteria and categorising evidence as did the EULAR taskforce. Results: A total of 44 articles and 10 abstracts were identified fulfilling the eligibility and exclusion criteria. Strong new evidence exists for recommendation 3 with nurse-led telephone services to enhance continuity of care and to provide ongoing support (evidence level 3), and - at least in part - for recommendation 6 , that nurses should promote self-efficacy (evidence level 1B) and empowerment (evidence level 2B), but sense of control was not studied. Some new evidence also exists for recommendations 7 and 8 (level 2B).
Conclusions: This literature review reveals new evidence for a role of nurses in managing CIA-patients especially with RA and in stable and low disease activity, and thus further supports the existing 2011 EULAR-recommendations.

\section{REFERENCES}

[1] van Eijk-Hustings $Y$, van Tubergen A, Boström C, et al. (2012) EULAR recommendations for the role of the nurse in the management of chronic inflammatory arthritis. Ann Rheum Dis 71:13-19

[2] van Eijk-Hustings Y, Ndosi M, Buss B, et al. (2014) Dissemination and evaluation of the European League Against Rheumatism recommendations for the role of the nurse in the management of chronic inflammatory arthritis: results of a multinational survey among nurses, rheumatologists and patients. Rheumatology 53:1491-96

Acknowledgements:

Disclosure of Interest: None declared

DOI: 10.1136/annrheumdis-2018-eular.3708

\section{AB1273 UPTAKE OF PNEUMOCOCCAL AND INFLUENZA VACCINATION IN PATIENTS RECEIVING BIOLOGICAL DMARDS (BDMARDS) IN IRELAND}

W.L. $\mathrm{Ng}^{1,2}$, A. Anjum ${ }^{1,2}$, A. Sebastian ${ }^{1,2}$, M. Brady ${ }^{1,2}$, E. Fitzgerald ${ }^{2}$, B. McCarthy ${ }^{2}$, M. Gillespie ${ }^{1}$, J.P. Doran ${ }^{1}$, J. Devlin ${ }^{1,2}$, A. Fraser ${ }^{1,2}$. ${ }^{1}$ Rheumatology, University Hospital Limerick: ${ }^{2}$ Rheumatology, Croom Orthopaedic Hospital, Limerick, Ireland

Background: Biological disease-modifying antirheumatic drugs (bDMARDs) have made significant positive outcomes in the lives of patients with rheumatic disease. This treatment has proven efficacy in delaying joint destruction and inducing disease remission. ${ }^{1}$ Studies have shown that pneumococcal vaccination is cost effective while the influenza vaccination significantly prevents morbidity and mortality in the elderly and in patients with chronic disease.

Objectives: To evaluate the pneumococcal and influenza vaccination status in patients receiving biological disease-modifying antirheumatic drugs (bDMARDs). Methods: Patients on bDMARDs attending the rheumatology infusion unit were asked about their vaccination status on pneumococcal and influenza using a questionnaire. The patients' diagnosis, current bDMARD and reasons for not having had vaccination were recorded.

Results: 92 patients were recruited. Mean age of 53.2 years with $63(68.5 \%)$ female and $29(31.5 \%)$ male. A total of $30(32.6 \%)$ patients received both pneumococcal and influenza vaccination, $1(1.1 \%)$ received pneumococcal vaccination alone, $22(23.9 \%)$ received influenza vaccination alone and $39(42.4 \%)$ had neither. Of the $18(19.6 \%)$ patients age $>65$ years, $5(27.8 \%)$ received influenza vac cination alone and $8(44.4 \%)$ received both. Patients who did not receive vaccinations were given an educational booklet.

The most common diagnosis from our cohort was rheumatoid arthritis(37\%), followed by spondyloarthritis(13\%), Behçet's disease(9.8\%), myositis $(7.6 \%)$, vasculitis(5.4\%), systemic lupus erythematosus(5.4\%), psoriatic arthritis(4.4\%) and others(17.4\%). 48 (52.2\%) were on rituximab, $37(40.2 \%)$ on infliximab, $6(6.5 \%)$ were on tocilizumab and $1(1.1 \%)$ was on abatacept.

Of the $61(66.3 \%)$ patients who did not receive the pneumococcal vaccine, 44 $(72.1 \%)$ were unaware of its availability, $6(9.8 \%)$ were not interested in receiving it, $4(6.6 \%)$ were afraid of the side effects, $4(6.6 \%)$ declined vaccination and 3 $(4.9 \%)$ were unaware it was recommended. $40(43.5 \%)$ who did not receive the influenza vaccine stated that they were either unaware $(45 \%)$, not interested $(25 \%)$, declined vaccination( $10 \%)$, forgotten $(5 \%)$, unaware it was recommended $(5 \%)$ and afraid of the side effects(2.5\%). $3(7.5 \%)$ had previous bad experiences from influenza vaccination.

Conclusions: This is the first study in Ireland looking at vaccination uptake in patients on bDMARDS. The vaccination rate in our cohort was less than satisfactory. Patients on immunosuppressants are recommended to have these vaccinations and preferably to receive them before commencing on the immunosuppressants. ${ }^{2}$ The lack of awareness is the main reason for failure to be vaccinated. Hence, primary care physicians and the rheumatology team should take active roles in increasing awareness amongst patients about the recommendation for pneumococcal and influenza vaccination.

\section{REFERENCES}

[1] Doe S, Pathare S, Kelly CA, et al. Uptake of influenza vaccination in patients on immunosuppressant agents for rheumatological diseases: a follow-up audit of the influence of secondary care. Rheumatology. 2007:46:715-6

\section{[] 2Assen}

S V, Agmon-Levin N, Elkayam O, et al. EULAR recommendations for vaccination in adult patients with autoimmune inflammatory rheumatic diseases. Ann Rheum Dis. 2011;70:414-22 